\title{
A Discrete Model for HIV Infection with Distributed Delay
}

\author{
Brahim EL Boukari, ${ }^{1}$ Khalid Hattaf, ${ }^{1,2}$ and Noura Yousfi ${ }^{1}$ \\ ${ }^{1}$ Department of Mathematics and Computer Science, Faculty of Sciences Ben M'sik, Hassan II University, \\ P.O. Box 7955 Sidi Othman, Casablanca, Morocco \\ ${ }^{2}$ Centre Régional des Métiers de l'Education et de la Formation (CRMEF), 20340 Derb Ghallef, Casablanca, Morocco
}

Correspondence should be addressed to Brahim EL Boukari; elboukaribrahim@yahoo.fr

Received 12 January 2014; Revised 9 April 2014; Accepted 9 April 2014; Published 27 April 2014

Academic Editor: Toka Diagana

Copyright (C) 2014 Brahim EL Boukari et al. This is an open access article distributed under the Creative Commons Attribution License, which permits unrestricted use, distribution, and reproduction in any medium, provided the original work is properly cited.

We give a consistent discretization of a continuous model of HIV infection, with distributed time delays to express the lag between the times when the virus enters a cell and when the cell becomes infected. The global stability of the steady states of the model is determined and numerical simulations are presented to illustrate our theoretical results.

\section{Introduction}

Nowadays, human immunodeficiency virus (HIV) that causes acquired immunodeficiency syndrome (AIDS) is a major health problem worldwide. From the World Health Organization (WHO), more than 35 million people are living with HIV/AIDS, and 1.6 million people died of this disease in 2012 [1]. Recent studies have been developed to know the dynamics of HIV infection, such as [2-9]. All these studies are based on continuous mathematical models. In reality, the statistical data are collected in discrete time, and the numerical simulations of continuous-time models are obtained by discretizing the models.

In this paper, we consider the model presented in [9] and we ignore the effect of the adaptive immune response. This model becomes as follows:

$$
\begin{aligned}
& \dot{x}(t)=\lambda-d x(t)-\beta x(t) v(t), \\
& \dot{y}(t)=\beta \int_{0}^{\theta} f(\tau) e^{-m \tau} x(t-\tau) v(t-\tau) d \tau-a y(t), \\
& \dot{v}(t)=k y(t)-\mu v(t),
\end{aligned}
$$

where $x(t), y(t)$, and $v(t)$ denote the concentration of uninfected cells, infected cells, and free virus particles at time $t$, respectively. The uninfected cells are produced at a constant $\lambda$, die at a rate $d x$, and become infected by free virus at a rate $\beta x v$. Infected cells are lost at a rate ay. Free viruses are produced by infected cells at a rate $k y$ and cleared at a rate $\mu \nu$. The authors [9] assumed that the uninfected cells are contacted by the virus particles at time $t-\tau$ and become infected cells at time $t$, where $\tau$ is a random variable with a probability distribution $f(\tau)$ over the interval $[0, \theta]$ and $\theta$ is limit superior of this delay. This probability distribution is assumed, for simplicity, to be a positive and integrable function on $[0, \theta]$, satisfying $\int_{0}^{\theta} f(\tau) d \tau=1$. The term $e^{-m \tau}$ is the probability of surviving from time $t-\tau$ to time $t$, where $m$ is the death rate of infected but not yet virus-producing cells.

Using the result presented in [9], it is not hard to see that the basic reproduction number of system (1) is given by $R_{0}^{*}=$ $(\lambda k \beta / a d \mu) \int_{0}^{\theta} f(\tau) e^{-m \tau} d \tau$.

We recall that the number $R_{0}^{*}$ is defined as the average number of secondary infections produced by one infected cell over its average life time, when all cells are uninfected.

In addition, the system (1) always has a disease-free equilibrium $E_{0}(\lambda / d, 0,0)$ which is globally asymptotically stable if $R_{0}^{*} \leq 1$ and a unique endemic equilibrium $E^{*}\left((\lambda / d)\left(1 / R_{0}^{*}\right),(\mu d / k \beta)\left(R_{0}^{*}-1\right),(d / \beta)\left(R_{0}^{*}-1\right)\right)$ is globally asymptotically stable when $R_{0}^{*}>1$.

Motivated by the works [10-15] and that the statistical data are collected in discrete time, we propose the following discrete model obtained from (1) by using the rectangle 
method to approximate the integral and by applying the backward Euler discretization:

$$
\begin{aligned}
& \frac{x_{n+1}-x_{n}}{h}=\lambda-d x_{n+1}-\beta x_{n+1} v_{n+1}, \\
& \frac{y_{n+1}-y_{n}}{h}=\beta \sum_{i=0}^{N} f(i) e^{-m i} x(n-i) v(n-i)-a y_{n+1}, \\
& \frac{v_{n+1}-v_{n}}{h}=k y_{n+1}-\mu v_{n+1},
\end{aligned}
$$

where $N=\theta-1$ if $\theta$ is an integer, and if not, $N$ is the integer part of $\theta(N=[\theta])$. The sequences $x_{n}, y_{n}$, and $v_{n}$ denote the concentration of uninfected cells, infected cells, and free virus particles at time $n$, respectively. The parameters in the system (2) are the same as those in (1). For simplicity, we may assume that $\sum_{i=0}^{N} f(i)=1$. Similar to the continuous system (1), system (2) always has a disease-free equilibrium $E_{0}(\lambda / d, 0,0)$ and an endemic equilibrium point $E^{*}\left((\lambda / d)\left(1 / R_{0}\right),(\mu d / k \beta)\left(R_{0}-1\right),(d / \beta)\left(R_{0}-1\right)\right)$, where $R_{0}$ is the basic reproduction number of (2) which is defined by

$$
R_{0}=\frac{\lambda k \beta}{a d \mu} \sum_{i=0}^{N} f(i) e^{-m i} .
$$

The aim of this work is to show that the discretization scheme used in system (2) preserves the positivity and boundedness of solutions and the global stability of both equilibria for the continuous model (1). Therefore, this discretization is dynamically consistent which means that all of the critical, qualitative properties of the solutions to the system of differential equations should also be satisfied by the solutions of the discrete scheme.

The paper is organized as follows. Section 2 deals with positivity and boundedness of solutions. In Section 3, we discuss the global stability of the equilibria. The numerical simulations are presented in Section 4 and the paper ends with a conclusion in Section 5.

\section{Positivity and Boundedness of Solutions}

Model (2) describes the evolution of a cell population that the cell number is nonnegative and bounded. For these biological reasons, we assume that the initial data for system (2) satisfy

$$
\begin{array}{r}
x(s) \geq 0, \quad y(s) \geq 0, \quad v(s) \geq 0 \\
\forall s=-N,-(N-1), \ldots, 0 .
\end{array}
$$

Proposition 1. All solution of system (2) subject to condition (4) remains nonnegative and bounded.

Proof. From (2), we have

$$
\begin{aligned}
x_{n+1} & =\frac{\lambda h+x_{n}}{1+d h+\beta h v_{n+1}} \\
y_{n+1} & =\frac{y_{n}+h \beta \sum_{i=0}^{N} f(i) e^{-m i} x(n-i) v(n-i)}{1+a h} \\
v_{n+1} & =\frac{v_{n}+h k y_{n+1}}{1+h \mu} .
\end{aligned}
$$

Hence, by recurrence and (4), we have $y_{n}$ nonnegative and, thereafter, $v_{n}$ and $x_{n}$ are nonnegative.

For the boundedness, we put $T_{n}=e^{m} x_{n}+y_{n}+$ $h \beta \sum_{i=0}^{N} f(i) \sum_{j=n-i}^{n} e^{-m(n-j)} x(j) v(j)$.

We have

$$
\begin{aligned}
T_{n+1}-T_{n}= & h e^{m}\left[\lambda-d x_{n+1}-\beta x_{n+1} v_{n+1}\right] \\
& +h\left[\beta \sum_{i=0}^{N} f(i) e^{-m i} x(n-i) v(n-i)-a y_{n+1}\right] \\
& +h \beta \sum_{i=0}^{N} f(i) \sum_{j=n+1-i}^{n+1} e^{-m(n+1-j)} x(j) v(j) \\
& -h \beta \sum_{i=0}^{N} f(i) \sum_{j=n-i}^{n} e^{-m(n-j)} x(j) v(j) \\
= & h\left[e^{m} \lambda-e^{m} d x_{n+1}-a y_{n+1}\right] \\
& +h\left[1-e^{m}\right] \beta \sum_{i=0}^{N} f(i) \sum_{j=n+1-i}^{n+1} e^{-m(n+1-j)} x(j) v(j) \\
\leq & h\left[\lambda e^{m}-\delta T_{n+1}\right],
\end{aligned}
$$

with $\delta=\inf \left\{d, a,\left(e^{m}-1\right) / h\right\}$. Then,

$$
\begin{aligned}
T_{n+1} & \leq\left(\frac{1}{1+h \delta}\right)^{(n+1)} T_{0}+\frac{\lambda e^{m}}{\delta}\left(1-\left(\frac{1}{1+h \delta}\right)^{(n+1)}\right) \\
& \leq T_{0}+\frac{\lambda e^{m}}{\delta} .
\end{aligned}
$$

Then, $x_{n}$ and $y_{n}$ are bounded.

By the third equation of (2), we have $v_{n+1}=(1 /(1+$ $h \mu)) v_{n}+(h k /(1+h \mu)) y_{n+1}$. Since $y_{n}$ is bounded, then there is $M$ such that $y_{n} \leq M$, for all $n \in \mathbb{N}$. Then, $v_{n+1} \leq(1 /(1+$ $h \mu)) v_{n}+(h k /(1+h \mu)) M$; hence, $v_{n+1} \leq(1 /(1+h \mu))^{n+1} v_{0}+$ $(k M / \mu)\left[1-(1 /(1+h \mu))^{n+1}\right] \leq v_{0}+(k M / \mu)$, and then $v_{n}$ is bounded.

\section{Global Stability}

In this section, we will give the following main result that characterizes the global behavior of our model.

\section{Theorem 2.}

(i) If $R_{0} \leq 1$, then $E_{0}$ is globally asymptotically stable.

(ii) $R_{0}>1$; then $E^{*}$ is globally asymptotically stable.

Proof. For (i), we consider the following sequence $\left\{U_{n}\right\}_{n=0}^{+\infty}$ defined by

$$
\begin{aligned}
U_{n}= & A x^{*} g\left(\frac{x_{n}}{x^{*}}\right)+y_{n} \\
& +h \beta \sum_{i=0}^{N} f(i) e^{-m i}\left[\sum_{j=0}^{i} x(n-j) v(n-j)\right]+\frac{a}{k} v_{n},
\end{aligned}
$$

with $A=\sum_{i=0}^{N} f(i) e^{-m i}, x^{*}=\lambda / d$, and $g(s)=s-1-\ln (s)$. 
It is clear that $g(s) \geq 0$ for any $s>0$ and $g$ has the global minimum $g(1)=0$.

Consider

$$
\begin{aligned}
& U_{n+1}-U_{n}= A x^{*}\left[\frac{x_{n+1}}{x^{*}}-\frac{x_{n}}{x^{*}}+\ln \left(\frac{x_{n}}{x_{n+1}}\right)\right]+y_{n+1}-y_{n} \\
&+h \beta \sum_{i=0}^{N} f(i) e^{-m i}[x(n+1) v(n+1) \\
&+\frac{a}{k}\left[v_{n+1}-v_{n}\right] \\
& \leq A\left[\left(x_{n+1}-x_{n}\right)\left(1-\frac{x^{*}}{x_{n+1}}\right)\right]+y_{n+1}-y_{n} \\
&+h \beta \sum_{i=0}^{N} f(i) e^{-m i}[x(n+1) v(n+1)] \\
&+\frac{a}{k}\left[v_{n+1}-v_{n}\right] \\
& \leq h A\left(1-\frac{x^{*}}{x_{n+1}}\right)\left[\lambda-d x_{n+1}-\beta x_{n+1} v_{n+1}\right] \\
&+h \beta \sum_{i=0}^{N} f(i) e^{-m i} x(n-i) v(n-i)-h a y_{n+1} \\
&+h \beta \sum_{i=0}^{N} f(i) e^{-m i}[x(n+1) v(n+1)] \\
&+h \frac{a}{k}\left[k y_{n+1}-\mu v_{n+1}\right] . \\
&-x(n-i) v(n-i)]
\end{aligned}
$$

Since $x^{*}=\lambda / d$, we have that

$$
\begin{aligned}
& U_{n+1}-U_{n} \\
& \leq h\left[-\frac{A}{x_{n+1}}\left(x_{n+1}-x^{*}\right)^{2}\right. \\
& \quad-A \beta x_{n+1} v_{n+1}+A \beta x^{*} v_{n+1} \\
& \quad+\beta \sum_{i=0}^{N} f(i) e^{-m i} x(n-i) v(n-i) \\
& \quad-\frac{a \mu}{k} v_{n+1}+\left(\beta \sum_{i=0}^{N} f(i) e^{-m i}\right) x(n+1) v(n+1) \\
& \left.\quad-\beta \sum_{i=0}^{N} f(i) e^{-m i}[x(n-i) v(n-i)]\right] \\
& \leq h\left[-\frac{A}{x_{n+1}}\left(x_{n+1}-x^{*}\right)^{2}-\left(1-R_{0}\right) \frac{a \mu}{k} v_{n+1}\right] \leq 0 .
\end{aligned}
$$

We consider the set $S=\left\{\left(x_{n}, y_{n}, v_{n}\right) \in \mathbb{R}_{+}^{3} / U_{n+1}-U_{n}=0\right\}$.
We have $\left(x_{n}, y_{n}, v_{n}\right) \in S \Rightarrow x_{n}=x^{*}$ and, by (2), we have $y_{n}=v_{n}=0$. By LaSalle's invariance principle (see [16, Theorem 4.24]), we have $E_{0}$ that is globally asymptotically stable.

For (ii), we consider the following sequence $\left\{w_{n}\right\}_{n=0}^{+\infty}$ defined by

$$
\begin{aligned}
w_{n}= & x^{*} g\left(\frac{x_{n}}{x^{*}}\right)+\frac{1}{A} y^{*} g\left(\frac{y_{n}}{y^{*}}\right)+\frac{\beta x^{*} v^{*}}{A} \\
& \times h \sum_{i=0}^{N} f(i) e^{-m i} \sum_{j=0}^{i} g\left(\frac{x(n-j) v(n-j)}{x^{*} v^{*}}\right) \\
& +\frac{a}{k A} v^{*} g\left(\frac{v_{n}}{v^{*}}\right),
\end{aligned}
$$

with $x^{*}=\lambda / d R_{0}, y^{*}=\mu d / k \beta\left(R_{0}-1\right)$, and $v^{*}=d / \beta\left(R_{0}-1\right)$.

Consider

$$
\begin{aligned}
& w_{n+1}-w_{n} \\
&= x^{*}\left[\frac{x_{n+1}-x_{n}}{x^{*}}+\ln \left(\frac{x_{n}}{x_{n+1}}\right)\right] \\
&+\frac{y^{*}}{A}\left[\frac{y_{n+1}-y_{n}}{y^{*}}+\ln \left(\frac{y_{n}}{y_{n+1}}\right)\right] \\
&+\frac{\beta x^{*} v^{*}}{A} h \sum_{i=0}^{N} f(i) e^{-m i} \\
& \times \sum_{j=0}^{i}\left(\frac{x(n+1-j) v(n+1-j)-x(n-j) v(n-j)}{x^{*} v^{*}}\right) \\
&+\frac{\beta x^{*} v^{*}}{A} h \\
& \times \sum_{i=0}^{N} f(i) e^{-m i} \sum_{j=0}^{i}\left(\ln \left(\frac{x(n-j) v(n-j)}{x(n+1-j) v(n+1-j)}\right)\right) \\
&+\frac{a v^{*}}{k A}\left[\frac{v_{n+1}-v_{n}}{v^{*}}+\ln \left(\frac{v_{n}}{v_{n+1}}\right)\right] \\
& \leq\left.\left(x_{n+1}-x_{n}\right)\left(1-\frac{x^{*}}{x_{n+1}}\right)\right] \\
&+\frac{1}{A}\left[\left(y_{n+1}-y_{n}\right)\left(1-\frac{y^{*}}{y_{n+1}}\right)\right] \\
&+ \frac{\beta x^{*} v^{*}}{A} h(i) e^{-m i} \sum_{j=0}^{i}\left(\ln \left(\frac{x(n-j) v(n-j)}{x(n+1-j) v(n+1-j)}\right)\right) \\
& x^{*} v^{*} v^{*}\left(\frac{x(n+1) v(n+1)-x(n-i) v(n)}{A} h\right.
\end{aligned}
$$




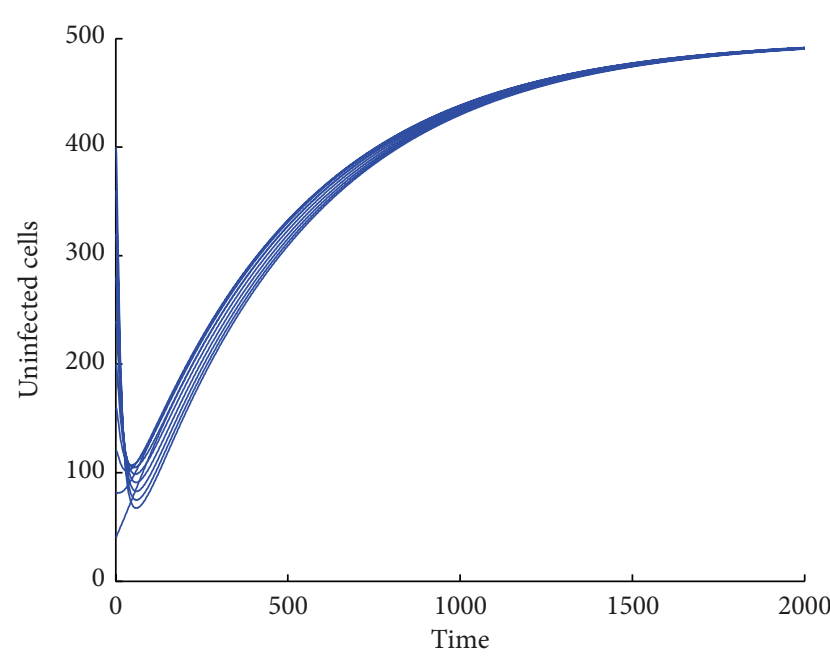

(a)

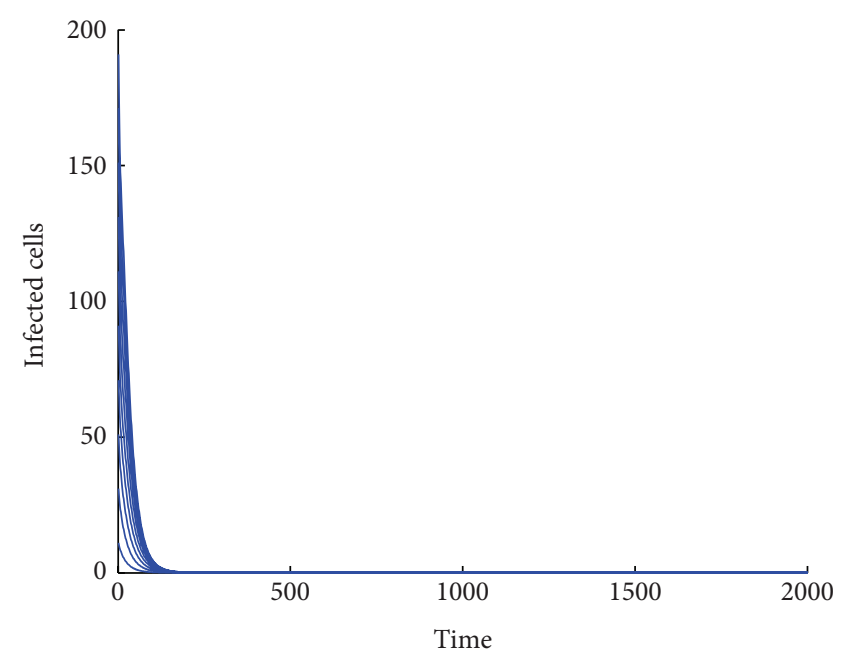

(b)

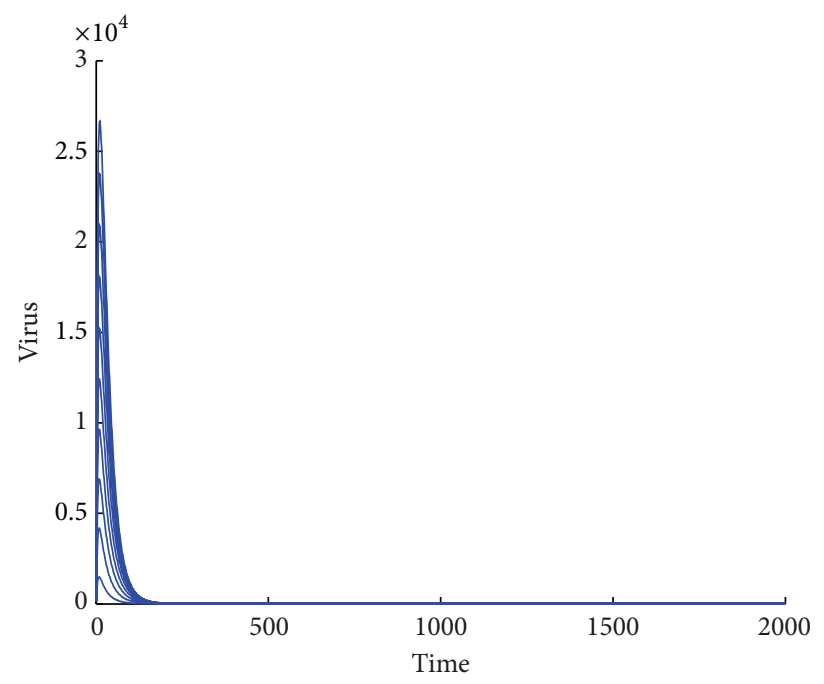

(c)

FIGURE 1: Demonstration of the stability of $E_{0}$.

$$
\begin{aligned}
& +\frac{a}{k A}\left[\left(v_{n+1}-v_{n}\right)\left(1-\frac{v^{*}}{v_{n+1}}\right)\right] \\
& \leq h\left[\left[\left(\lambda-d x_{n+1}-\beta x_{n+1} v_{n+1}\right)\left(1-\frac{x^{*}}{x_{n+1}}\right)\right]\right. \\
& +\frac{1}{A}\left[\left(\beta \sum_{i=0}^{N} f(i) e^{-m i} x(n-i) v(n-i)-a y_{n+1}\right)\right. \\
& \left.\quad \times\left(1-\frac{y^{*}}{y_{n+1}}\right)\right] \\
& +\beta x_{n+1} v_{n+1}-\frac{\beta x^{*} v^{*}}{A} \\
& \quad \times \sum_{i=0}^{N} f(i) e^{-m i}\left(\frac{x(n-i) v(n-i)}{x^{*} v^{*}}\right)
\end{aligned}
$$$$
+\frac{\beta x^{*} v^{*}}{A} \sum_{i=0}^{N} f(i) e^{-m i}\left(\ln \left(\frac{x(n-i) v(n-i)}{x(n+1) v(n+1)}\right)\right)
$$$$
\left.+\frac{a}{k A}\left[\left(k y_{n+1}-\mu v_{n+1}\right)\left(1-\frac{v^{*}}{v_{n+1}}\right)\right]\right] \text {. }
$$

Using the fact that $\lambda=d x^{*}+\beta x^{*} v^{*}$, we have

$$
\begin{aligned}
w_{n+1}-w_{n} & \\
\leq h[ & \frac{-d}{x_{n+1}}\left(x_{n+1}-x^{*}\right)^{2}+\beta x^{*} v^{*}-\beta x^{*} v^{*} \frac{x^{*}}{x_{n+1}} \\
& \quad-\frac{\beta y^{*}}{A y_{n+1}} \sum_{i=0}^{N} f(i) e^{-m i} x(n-i) v(n-i)+\frac{a}{A} y^{*}
\end{aligned}
$$




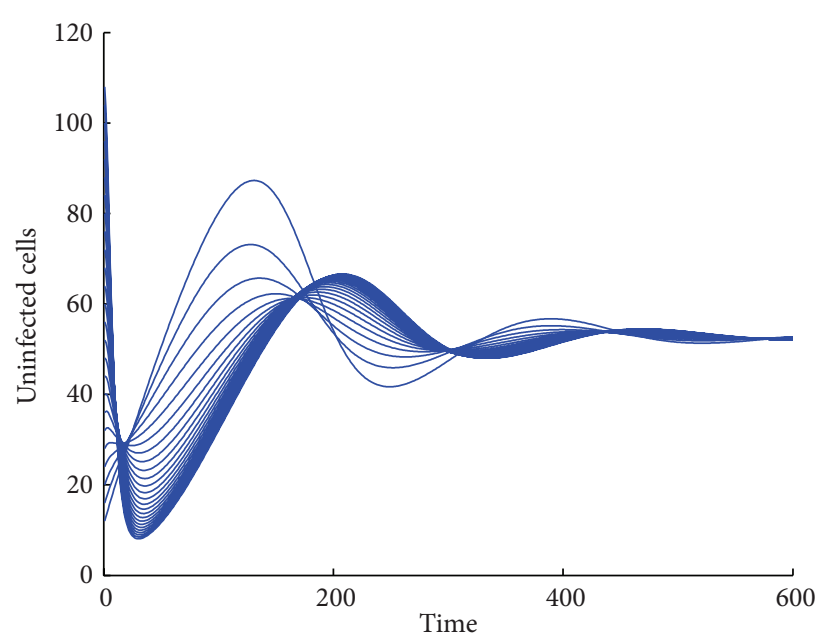

(a)

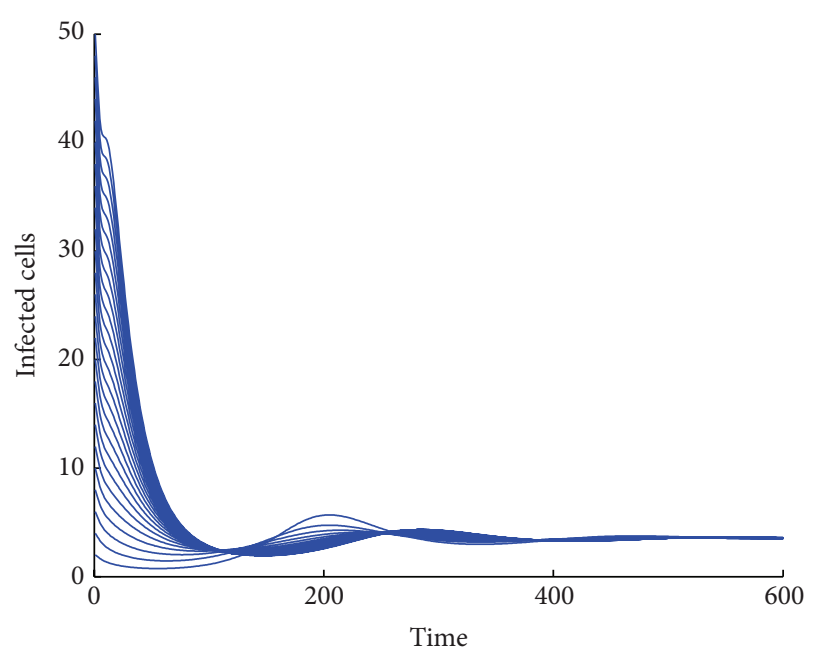

(b)

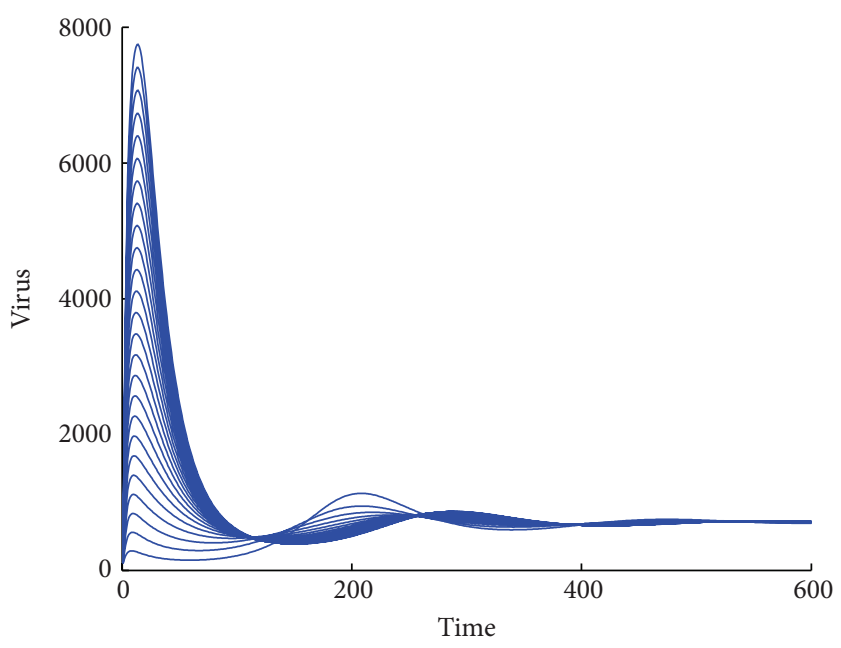

(c)

FIgURE 2: Demonstration of the stability of $E^{*}$.

$$
\begin{aligned}
& +\frac{\beta x^{*} v^{*}}{A} \sum_{i=0}^{N} f(i) e^{-m i}\left(\ln \left(\frac{x(n-i) v(n-i)}{x(n+1) v(n+1)}\right)\right) \\
& \left.+\frac{a}{k A}\left[\frac{-k v^{*} y_{n+1}}{v_{n+1}}+\mu v^{*}\right]\right] .
\end{aligned}
$$

Using the relations $\ln (x(n-i) v(n-i) / x(n+1) v(n+1))=$ $\ln \left(x(n-i) v(n-i) y^{*} / x^{*} v^{*} y(n+1)\right)+\ln \left(x^{*} / x(n+1)\right)+\ln \left(v^{*} y(n+\right.$ 1) $\left./ v(n+1) y^{*}\right), \beta A x^{*} v^{*}=a y^{*}$, and $k y^{*}=\mu v^{*}$, we obtain

$$
\begin{aligned}
w_{n+1}-w_{n} & \\
\leq h[ & \frac{-d}{x_{n+1}}\left(x_{n+1}-x^{*}\right)^{2}-\beta x^{*} v^{*} \\
\times & {\left[\frac{x^{*}}{x_{n+1}}-1-\ln \left(\frac{x^{*}}{x_{n+1}}\right)\right] } \\
& -\frac{\beta x^{*} v^{*}}{A}
\end{aligned}
$$

$$
\begin{gathered}
\times \sum_{i=0}^{N} f(i) e^{-m i}\left[\frac{x(n-i) v(n-i) y^{*}}{x^{*} v^{*} y_{n+1}}\right. \\
\left.-1-\ln \left(\frac{x(n-i) v(n-i) y^{*}}{x^{*} v^{*} y_{n+1}}\right)\right] \\
\left.-\beta x^{*} v^{*}\left[\frac{v^{*}}{y^{*}} \frac{y_{n+1}}{v_{n+1}}-1-\ln \left(\frac{v^{*}}{y^{*}} \frac{y_{n+1}}{v_{n+1}}\right)\right]\right] \\
\leq h\left[\frac{-d}{v_{n+1}}\left(x_{n+1}-x^{*}\right)^{2}-\beta x^{*} v^{*} g\left(\frac{x^{*}}{x_{n+1}}\right)\right. \\
-\frac{\beta x^{*} v^{*}}{A} \sum_{i=0}^{N} f(i) e^{-m i} g\left(\frac{x(n-i) v(n-i) y^{*}}{x^{*} v^{*} y_{n+1}}\right) \\
\left.-\beta x^{*} v^{*} g\left(\frac{v^{*}}{y^{*}} \frac{y_{n+1}}{v_{n+1}}\right)\right]
\end{gathered}
$$




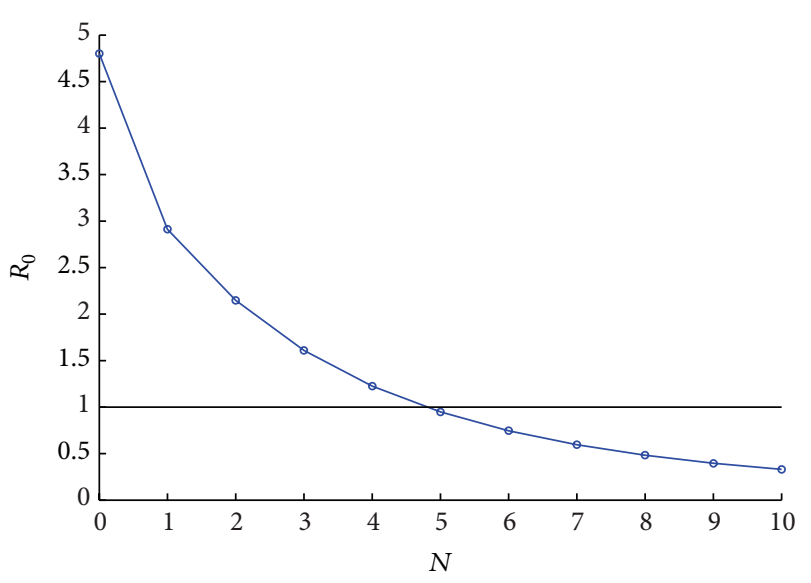

Figure 3: Plot of the basic reproduction number $R_{0}$ as a function of the delay $N$. Here, $\lambda=10, d=0.02, \beta=0.000024, m=0.5, a=0.5$, $k=600, \mu=3$, and $h=0.1$.

We consider the set $T=\left\{\left(x_{n}, y_{n}, v_{n}\right) \in \mathbb{R}_{+}^{3} / w_{n+1}-w_{n}=0\right\}$. We have $\left(x_{n}, y_{n}, v_{n}\right) \in T \Rightarrow x_{n}=x^{*}$ and, by (2), we have $y_{n}=y^{*}$ and $v_{n}=v^{*}$. From LaSalle's invariance principle, we deduce that $E^{*}$ is globally asymptotically stable.

\section{Numerical Simulations}

In this section, we present the numerical simulations to illustrate our theoretical results. In this section, we choose $f(x)=2 x / N(N+1)$. First, we use the following data set: $\lambda=10, d=0.02, \beta=0.000024, m=0.5$, $a=0.5, k=600, \mu=3, N=5$, and $h=0.1$. In this case, the basic infection reproduction number $R_{0}$ is 0.9483 . By using Theorem 2 (i), we deduce that $E_{0}$ is globally asymptotically stable. Numerical simulation illustrates our result (see Figure 1).

In Figure 2, we choose $\beta=0.00024$ and do not change the other parameter values. By calculation, we have $R_{0}=$ 9.4830 which satisfies the condition (ii) of Theorem 2. Hence, $E^{*}$ is globally asymptotically stable. Numerical simulation illustrates our result (see Figure 2).

In Figure 3, the parameter values are the same as those in Figure 1. Figure 3 gives $R_{0}$ in function of $N$ and shows that the growth of $N$ decreases the value of $R_{0}$ below 1, making the disease-free equilibrium globally asymptotically stable.

\section{Conclusion}

In this work, we have proposed a discrete mathematical model of HIV infection by applying the backward Euler discretization, with distributed time delay. We have proved that, when $R_{0} \leq 1$, the disease-free equilibrium $E_{0}$ is globally asymptotically stable. When $R_{0}>1$, the endemic equilibrium $E^{*}$ is globally asymptotically stable. More precisely, it is proved that this discretization guarantees the correct dynamic behavior regardless of the size of the time step.

\section{Conflict of Interests}

The authors declare that there is no conflict of interests regarding the publication of this paper.

\section{Acknowledgments}

The authors would like to thank the editor and the anonymous referees for their valuable comments and suggestions that have led to improvement of the quality of this work.

\section{References}

[1] WHO, Global Health Observatory (GHO), http://www.who. int/gho/hiv/en/.

[2] P. W. Nelson and A. S. Perelson, "Mathematical analysis of delay differential equation models of HIV-1 infection," Mathematical Biosciences, vol. 179, no. 1, pp. 73-94, 2002.

[3] R. Xu, "Global dynamics of an HIV-1 infection model with distributed intracellular delays," Computers \& Mathematics with Applications, vol. 61, no. 9, pp. 2799-2805, 2011.

[4] B. EL Boukari, K. Hattaf, and N. Yousfi, "Modeling the therapy of HIV infection with CTL response and cure rate," International Journal of Ecological Economics and Statistics, vol. 28, no. 1, pp. 1-17, 2013.

[5] K. Hattaf and N. Yousfi, "Dynamics of HIV infection model with therapy and cure rate," International Journal of Tomography and Statistics, vol. 16, no. 11, pp. 74-80, 2011.

[6] K. Hattaf and N. Yousfi, "Optimal control of a delayed HIV infection model with immune response using an efficient numerical method," ISRN Biomathematics, vol. 2012, Article ID 215124, 7 pages, 2012.

[7] K. Hattaf and N. Yousfi, "Two optimal treatments of HIV infection model," World Journal of Modelling and Simulation, vol. 8, pp. 27-35, 2012.

[8] K. Hattaf and N. Yousfi, "A delay differential equation model of HIV with therapy and cure rate," International Journal of Nonlinear Science, vol. 12, no. 4, pp. 503-512, 2011.

[9] B. EL Boukari, K. Hattaf, and N. Yousfi, "HIV infection model with immunity and distributed delay," Applied Mathematical Sciences, vol. 8, no. 14, pp. 653-661, 2014.

[10] Y. Enatsu, Y. Nakata, and Y. Muroya, "Global stability for a class of discrete SIR epidemic models," Mathematical Biosciences and Engineering, vol. 7, no. 2, pp. 347-361, 2010.

[11] Y. Enatsu, Y. Nakata, Y. Muroya, G. Izzo, and A. Vecchio, "Global dynamics of difference equations for SIR epidemic models with a class of nonlinear incidence rates," Journal of Difference Equations and Applications, vol. 18, no. 7, pp. 1163-1181, 2012.

[12] G. Izzo and A. Vecchio, "A discrete time version for models of population dynamics in the presence of an infection," Journal of Computational and Applied Mathematics, vol. 210, no. 1-2, pp. 210-221, 2007.

[13] R. E. Mickens, Advances in the Applications of Nonstandard Finite Difference Schemes, World Scientific Publishing Co. Pte. Ltd., Hackensack, NJ, USA, 2005.

[14] S. Elaydi and R. J. Sacker, "Global stability of periodic orbits of non-autonomous difference equations and population biology," Journal of Differential Equations, vol. 208, no. 1, pp. 258-273, 2005. 
[15] S. Jang and S. Elaydi, "Difference equations from discretization of a continuous epidemic model with immigration of infectives," Mathematics Faculty Research, paper 32, 2004.

[16] S. Elaydi, An Introduction to Difference Equations, Springer, New York, NY, USA, 3rd edition, 2005. 


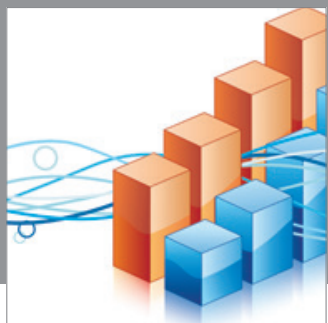

Advances in

Operations Research

mansans

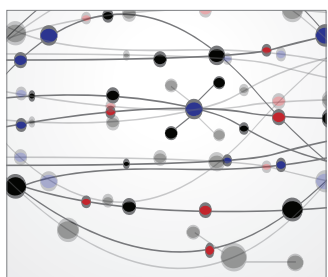

The Scientific World Journal
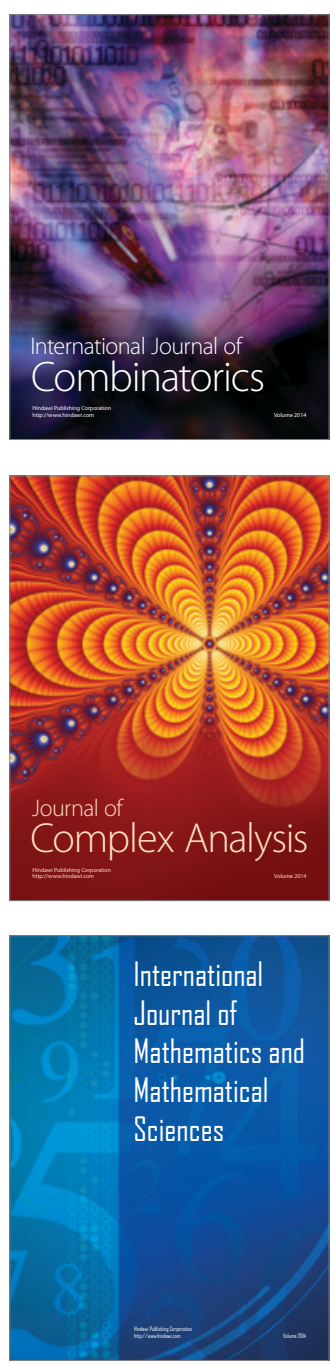
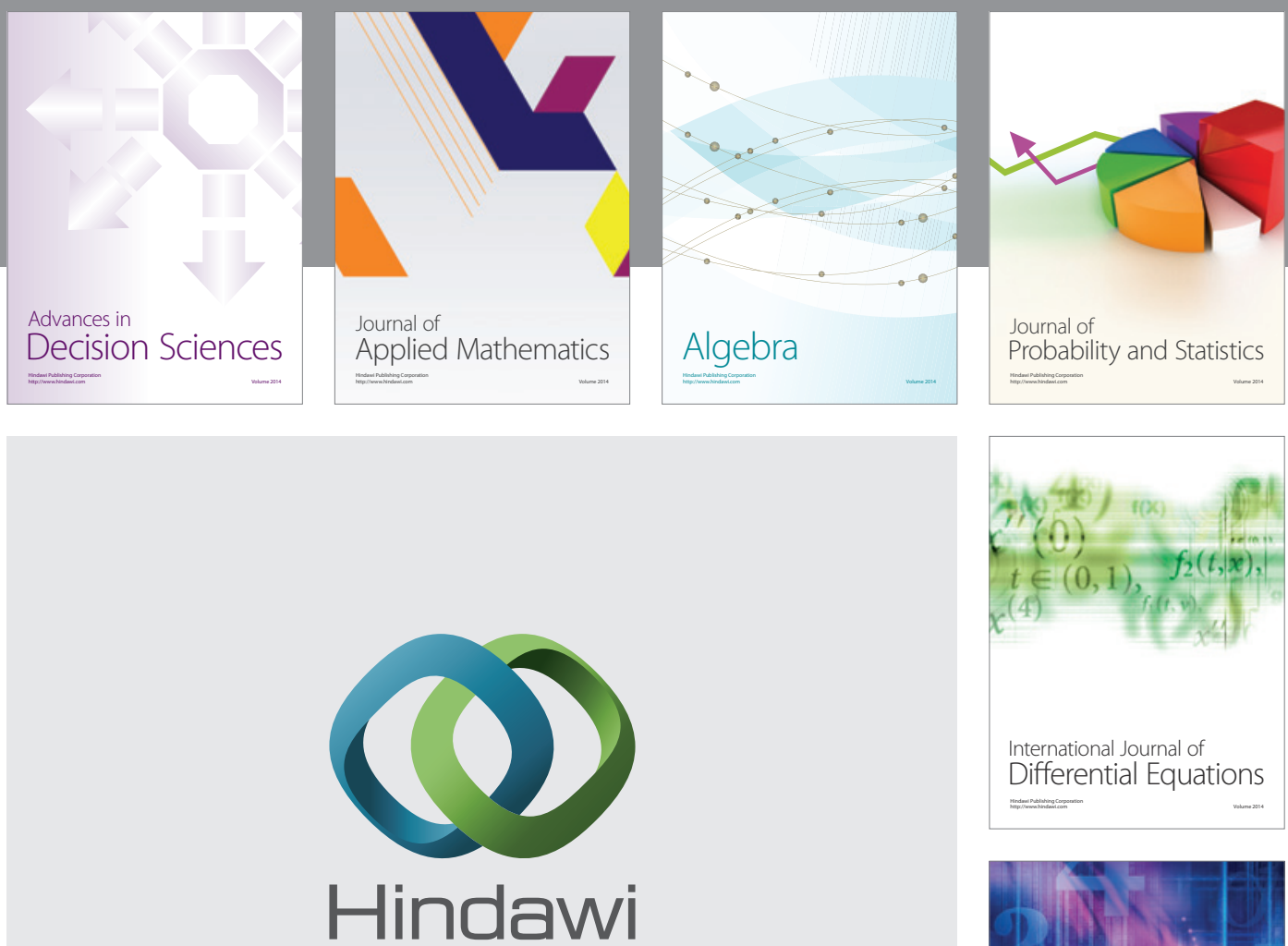

Submit your manuscripts at http://www.hindawi.com
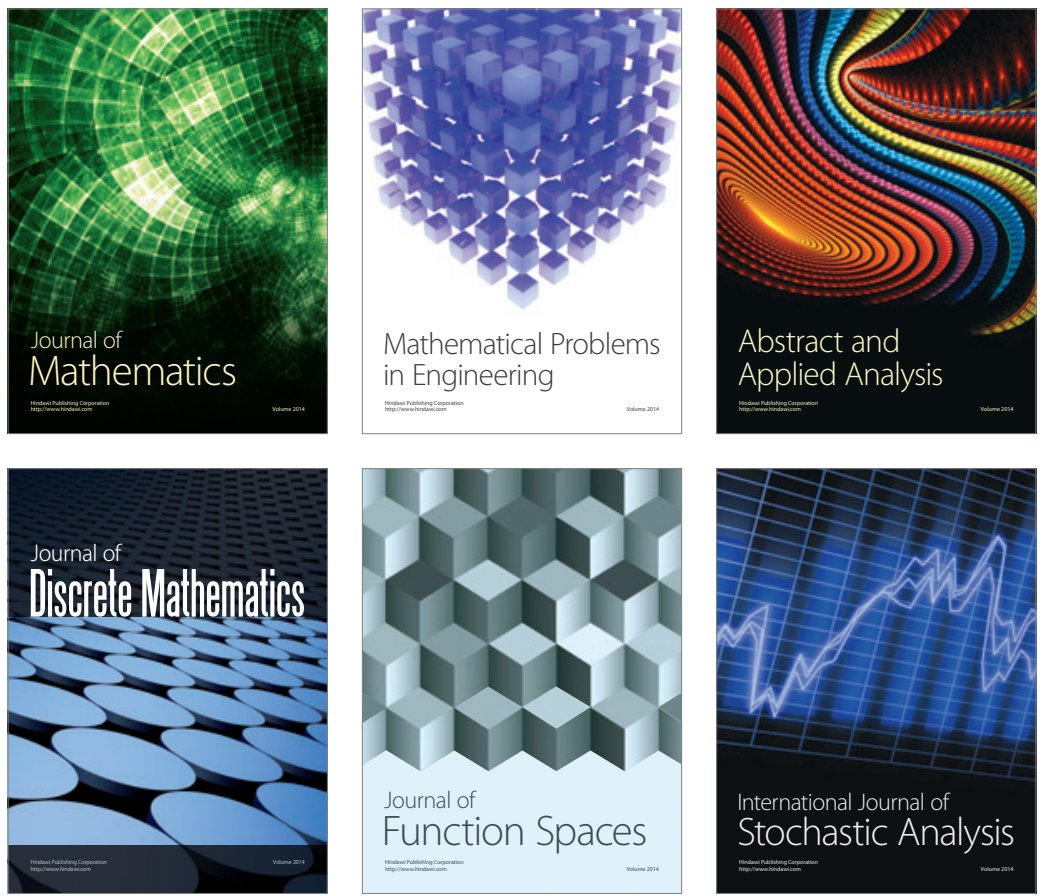

Journal of

Function Spaces

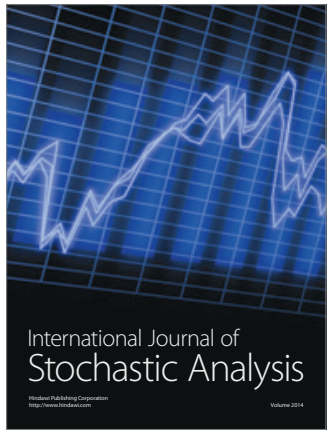

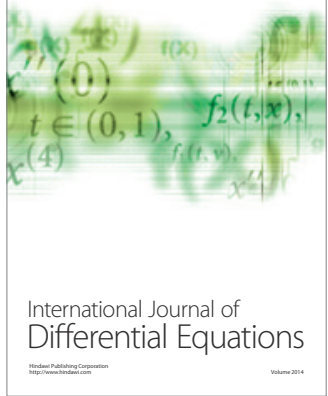
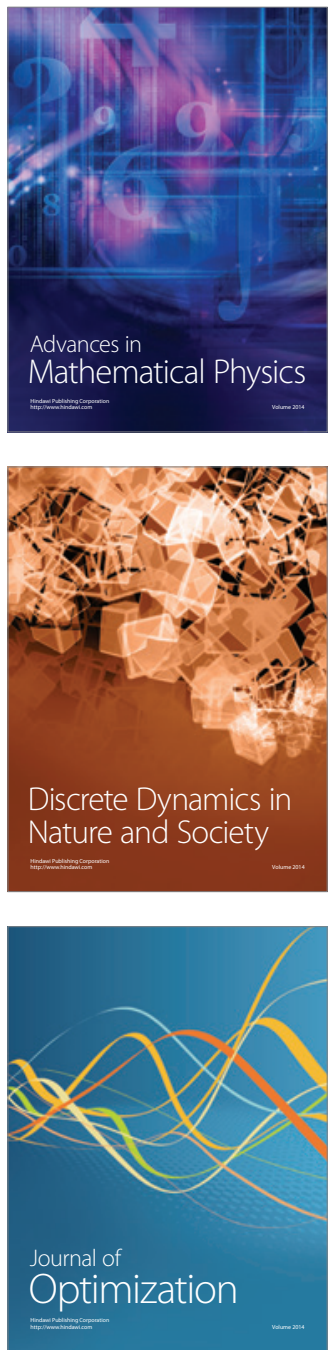